\title{
VESTIBOLOGY
}

\section{MR imaging of endolymphatic hydrops in Ménière's disease: not all that glitters is gold}

\author{
MR imaging dell'idrope endolinfatica nella malattia di Ménière: \\ non è oro tutto quel che luccica
}

\author{
G. Conte', F. M. Lo Russo², S. F. Calloni², C. Sina', S. Barozzi ${ }^{3}$, F. Di Berardino ${ }^{3,4}$, E. Scola', \\ G. Palumbo ${ }^{1}$, D. Zanetti ${ }^{4}$, F. M. Triulzi $i^{1,5}$ \\ ${ }^{1}$ Neuroradiology Unit, Fondazione IRCCS Ca' Granda Ospedale Maggiore Policlinico, Milan, Italy; ${ }^{2}$ Postgraduation \\ School in Radiodiagnostics, Università degli Studi di Milano, Milan, Italy; ${ }^{3}$ Department of Clinical Sciences and \\ Community Health, Università degli Studi di Milano, Milan, Italy; ${ }^{4}$ Audiology Unit, Fondazione IRCCS Ca'Granda \\ Ospedale Maggiore Policlinico, Milan, Italy; ${ }^{5}$ Department of Pathophysiology and Transplantation, Università degli \\ Studi di Milano, Milan, Italy
}

\section{SUMMARY}

Ménière's disease (MD) is a chronic condition characterised by fluctuating hearing loss, intermittent vertigo, tinnitus and aural fullness. Its anatomical and pathological counterpart is represented by endolymphatic hydrops (EH). Recent development and progress in magnetic resonance (MR) imaging techniques has enabled visualisation of EH in living human subjects using a 3 Tesla (T) scanner and gadoliniumbased contrast-agent (GBCA) via intravenous (IV) or intra-tympanic (IT) administration. Data emerging from the literature about MR imaging of EH in MD patients are limited, and we therefore reviewed the most common MR imaging findings in the study of the endolymphatic space in both MD and non-MD patients.

KEY WORDS: Magnetic resonance imaging • Endolymphatic hydrops • Ménière's disease • Cochlea • Vestibule

\section{RIASSUNTO}

La malattia di Ménière è una condizione cronica caratterizzata da sordità, vertigini, acufeni e sensazione di aumento della pressione intra auricolare. La sua controparte anatomo-patologica è l'idrope endolinfatica. I recenti progressi in campo di imaging di risonanza magnetica (RM) hanno permesso di visualizzare la presenza di idrope endolinfatica in vivo mediante l'acquisizione di immagini su scanner 3 Tesla dopo la somministrazione di mezzo di contrasto per via endovenosa o intratimpanica. I recenti dati di letteratura sull'imaging RM della sindrome di Ménière e la caratterizzazione dell'idrope endolinfatica sono tuttavia contradditori. Obiettivo di questo lavoro è la revisione dei reperti radiologici RM più comuni nello studio dell'idrope endolinfatica in pazienti affetti e non affetti da sindrome di Ménière.

PAROLE CHIAVE: Imaging di Risonanza Magnetica $\bullet$ Idrope endolinfatica $\bullet$ Malattia di Ménière $\bullet$ Coclea $\bullet$ Vestibolo

Acta Otorhinolaryngol Ital 2018;38:369-376

\section{Introduction}

Ménière's disease (MD) is a chronic condition characterised by fluctuating hearing loss, intermittent vertigo, tinnitus and aural fullness. It is a relatively common disorder, with a prevalence of $200-500$ per $100,000{ }^{1}$. Its anatomical and pathological counterpart is represented by endolymphatic hydrops (EH), a distension of the endolymphatic space of the inner ear into areas that are normally occupied by the perilymphatic space. The most common affected areas are the cochlear duct and the saccule, but EH may also involve the utriculum and semicir- cular canals ${ }^{2}$. Guidelines for diagnosis of this syndrome were established in 1995 by the American Academy of Otolaryngology - Head and Neck Surgery (AAO-HNS). The most recent and revised classification considers two major categories: definite and probable Ménière's disease $^{3}$. Diagnosis of definite MD is made by the presence of two or more episodes of vertigo, audiometrically documented low-to-medium frequency sensorineural hearing loss in one hear, and fluctuating aural fullness. Probable MD is defined by two or more episodes of vertigo, and fluctuating aural fullness in the affected ear ${ }^{4}$. To evalu- 
ate the presence of $\mathrm{EH}$, both electronystagmography and electrocochleography tests can be used, which reflect the reduced vestibular response and the elevation of inner ear pressure through distension of the basilar membrane, respectively. An emerging technique in assessment of $\mathrm{EH}$ is vestibular evoked myogenic potential (VEMP), a neuroelectrophysiological test that evaluates the otolithic organs of the utricle and saccule. VEMP may be abolished in patients with $\mathrm{EH}$ in the vestibule. Imaging studies in the past were mainly used to exclude retro cochlear disorder, such as schwannoma. However, the recent development and progress of magnetic resonance (MR) imaging techniques has enabled visualizsation of $\mathrm{EH}$ in living human subjects using a 3 Tesla (T) scanner and gadolinium-based contrast-agent (GBCA) via intravenous (IV) or intratympanic (IT) administration ${ }^{56}$. Data from the literature about MR imaging of EH in MD patients are limited due to discrepancies in patient selection and MR assessment criteria. For this reason, our purpose is to provide insight of the current MD imaging scenario. We reviewed the main techniques in assessment of EH and most common MR imaging findings in study of the endolymphatic space in both MD and non-MD patients.

\section{MR findings in MD patients}

In June 2017, a structured search was performed in PubMed using the following key words: "MRI" AND "endolymphatic hydrops" ( $=153)$, "MRI" AND "Ménière's disease" $(n=213)$. The search was filtered for studies on human subjects and published in English language. In order to have a clearer view of the potential diagnostic role of MR imaging in the assessment of patients with MD, we analysed only MR findings of studies on patients with definite MD according to the AAO-HNS criteria, who underwent MR imaging at 3 Tesla scanner with IT or IV administration of GBCA and volumetric acquisition. We excluded from the review: 1) case reports, reviews and metanalysis; 2) studies on patients enrolled according to diagnostic criteria other than those of the AAO-HNS, or in which clinical evidence of diagnosis ("definite", "probable" or "possible") according to those criteria was not specified; 3) studies in which the hydrops was not assessed by MR standardised qualitative, quantitative or semi-quantitative methods; 4) studies in which a per-ear analysis (symptomatic ear versus asymptomatic ear) of MR findings was not performed for each subject.

After the titles and abstracts of preliminary articles were read, 18 articles were deemed eligible. Two readers (F.L. and S.C., radiology residents with 1-3 years of experience in otoradiology, respectively) then read the articles in their entirety, confirming the eligibility for 17 of them.
They subsequently extracted the following information from eligible studies: first author, total number of subjects (MD patients and controls), MR acquisition and analysis methods and MR findings of particular interest for the evaluation of the hydrops in the symptomatic/asymptomatic ears of the enrolled subjects.

Results are reported separately for studies adopting ITGBCA administration and those adopting IV-GBCA administration. The results of the search are summarised in Tables I and II.

\section{Results and discussion}

\section{MR technique}

MR assessment of the endolymphatic space can be performed using both IT and IV administration of GBCA ${ }^{7}$. The IT-GBCA technique consists in IT administration of 0.3-0.6 $\mathrm{ml}$ of GBCA, diluted 8-fold, into the tympanic cavity by puncture of the tympanic membrane. The contrast medium diffuses into the perilymph, but not in the endolymph, depending on the permeability of the round window, giving a perilymph positive image (PPI) ${ }^{8}$. MR imaging is usually performed at 24 hours after administration, and a heavily T2-weighted 3D-FLAIR sequence with variable flip angle is usually preferred. The endolabyrinth appears to have a lower signal compared to the surrounding perilymph. The inversion time of the 3D-FLAIR can also be shortened to suppress the signal of the perilymph and increase that of the endolymph, thus giving a positive endolymphatic image (PEI) ${ }^{9}$. 3D inversion-recovery turbo spin-echo with real reconstruction (3D-real IR) allows to separate the signals from the perilymph (positive), endolymph (negative) and surrounding bone (zero) using an inversion time between the null point of the perilymph containing the contrast medium and the endolymph. However, this sequence is less sensitive to low GBCA concentrations than 3D-FLAIR 9.

The IV-GBCA technique consists in IV administration of a recommended dose of GBCA (usually 0.1 or $0.2 \mathrm{ml} / \mathrm{Kg}$ ) that slowly accumulates in the perilymph, but not in the endolymph, depending on the permeability of the blood-labyrinthine barrier, thus giving a PPI ${ }^{10}$. Two types of sequences are recommended: 3D-real IR or heavily T2-weighted 3DFLAIR ${ }^{7}$. The optimal time for MR acquisition is about 4 hours from the contrast-medium administration as demonstrated in another study concerning various fluid-containing spaces ${ }^{10}$. It is important to note that the visibility of the endolymphatic space depends on the inversion time. For this reason, it is recommended to acquire MR images in control subjects, in order to establish the normal standard for these sequences. MR images can be directly evaluated after acqui- 
Table I. MR findings for IV-GBCA technique.

\begin{tabular}{|c|c|c|c|c|c|c|}
\hline Articles & $\begin{array}{l}\text { MR sequence } \\
\text { (TR/TI/TE) }\end{array}$ & $\begin{array}{l}\text { Reference MR criteria } \\
\text { for the assessment } \\
\text { of EH }\end{array}$ & $\begin{array}{l}\text { Percentage (\%) } \\
\text { of EH } \\
\text { in symptomatic } \\
\text { MD ear }\end{array}$ & $\begin{array}{l}\text { Percentage (\%) } \\
\text { of EH } \\
\text { in asymptomatic } \\
\text { MD ear }\end{array}$ & $\begin{array}{l}\text { Percentage (\%) } \\
\text { of EH in ears with } \\
\text { other audiological } \\
\text { disorders }\end{array}$ & $\begin{array}{l}\text { Percentage (\%) } \\
\text { of EH } \\
\text { in healthy ears }\end{array}$ \\
\hline $\begin{array}{l}\text { Pakdamn } \\
\text { et al }{ }^{19}\end{array}$ & $\begin{array}{c}\text { 3D-FLAIR } \\
(9000 / 2350 / 534)\end{array}$ & $\begin{array}{l}\text { VEH if VES/vestibule > 50\% } \\
\text { cEH: not investigated }\end{array}$ & vEH: 22/32 (68\%) & vEH: 0/32 (0\%) & vEH: 0/11 (0\%) & vEH: 0/11 (0\%) \\
\hline $\begin{array}{l}\text { Sano } \\
\text { et al. }{ }^{15}\end{array}$ & $\begin{array}{c}\text { 3D-FLAIR } \\
(9000 / 2400 / 540)\end{array}$ & $\begin{array}{l}\text { vEH if VES/vestibule }>33 \% \\
\text { cEH if cochlear duct is dilatated }\end{array}$ & $\begin{array}{l}\text { vEH 1/1 (100\%) } \\
\text { cEH: } 1 / 1(100 \%)\end{array}$ & $\begin{array}{l}\text { vEH: } 1 / 1(100 \%) \\
\text { cEH: } 1 / 1(100 \%)\end{array}$ & $\begin{array}{l}\text { vEH: 4/6 (66\%) } \\
\text { cEH: 4/6 (66\%) }\end{array}$ & $\begin{array}{l}\text { vEH: } 2 / 4(50 \%) \\
\text { cEH: 0/4 (0\%) }\end{array}$ \\
\hline $\begin{array}{l}\text { Barath } \\
\text { et al. }{ }^{21}\end{array}$ & $\begin{array}{c}\text { 3D-real IR } \\
(6000 / 2000 / 177)\end{array}$ & $\begin{array}{l}\text { vEH if VES/vestibule }>50 \% \\
\text { cEH if cochlear duct is dilatated }\end{array}$ & EH: 41/43 (95\%) & EH: 10/45 (22\%) & NA & NA \\
\hline $\begin{array}{l}\text { Sepahdari } \\
\text { et al. }{ }^{13}\end{array}$ & $\begin{array}{c}\text { 3D-FLAIR } \\
(9000 / 2350 / 534)\end{array}$ & $\begin{array}{c}\text { vEH if VES/vestibule > } 45 \% \\
\text { cEH: not reported }\end{array}$ & vEH: 6/12 (50\%) & NA & NA & NA \\
\hline $\begin{array}{l}\text { Yoshida } \\
\text { et al. }{ }^{16}\end{array}$ & $\begin{array}{l}\text { 3D-FLAIR } \\
\text { (not specified) }\end{array}$ & $\begin{array}{l}\text { vEH if VES/vestibule }>33 \% \\
\text { cEH if cochlear duct is dilatated }\end{array}$ & $\begin{array}{l}\text { vEH: 49/52 (94\%) } \\
\text { cEH: 45/52 (86\%) }\end{array}$ & $\begin{array}{l}\text { VEH; } 17 / 32(53 \%) \\
\text { cEH: } 15 / 32(46 \%)\end{array}$ & NA & $\begin{array}{l}\text { vEH: } 3 / 42(7 \%) \\
\text { cEH: } 16 / 42(33 \%)\end{array}$ \\
\hline $\begin{array}{l}\text { Tagaya } \\
\text { et al. }{ }^{17}\end{array}$ & $\begin{array}{l}\text { 3D-FLAIR } \\
\text { (not specified) }\end{array}$ & $\begin{array}{l}\text { vEH if VES/vestibule }>33 \% \\
\text { cEH if cochlear duct is dilatated }\end{array}$ & $\begin{array}{l}\text { vEH: } 7 / 7(100 \%) \\
\text { cEH: } 5 / 7(71 \%)\end{array}$ & $\begin{array}{l}\text { vEH: } 3 / 5(60 \%) \\
\text { cEH: } 1 / 5(20 \%)\end{array}$ & NA & \\
\hline $\begin{array}{l}\text { Attyè } \\
\text { et al. }{ }^{18}\end{array}$ & $\begin{array}{c}\text { 3D-FLAIR } \\
(7600 / 2300 / 345)\end{array}$ & $\begin{array}{l}\text { vEH if VES/vestibule }>50 \% \\
\text { cEH if cochlear duct is dilatated }\end{array}$ & $\begin{array}{l}\text { vEH: } 14 / 30(46 \%) \\
\text { cEH: } 11 / 30(36 \%)\end{array}$ & NA & NA & $\begin{array}{l}\text { vEH: } 9 / 30(30 \%) \\
\text { cEH: } 4 / 30(13 \%)\end{array}$ \\
\hline $\begin{array}{l}\text { Sepahdari } \\
\text { et al. }{ }^{22}\end{array}$ & $\begin{array}{c}\text { 3D-FLAIR } \\
(9000 / 2350 / 534)\end{array}$ & $\begin{array}{l}\text { vEH if VES/vestibule }>50 \% \\
\text { cEH if cochlear duct is dilatated }\end{array}$ & EH: 7/7 (100\%) & EH: 0/7 (0\%) & NA & NA \\
\hline $\begin{array}{l}\text { Attyè } \\
\text { et al. }{ }^{20}\end{array}$ & $\begin{array}{c}\text { 3D-FLAIR } \\
(8000 / 2400 / 316)\end{array}$ & $\begin{array}{l}\text { vEH if VES/vestibule }>50 \% \\
\text { cEH if cochlear duct is dilatated }\end{array}$ & EH: 73/95 (77\%) & EH: 9/41 (21\%) & $\begin{array}{l}\text { vEH: } 15 / 128(11 \%) \\
\text { cEH: } 31 / 128(24 \%)\end{array}$ & NA \\
\hline
\end{tabular}

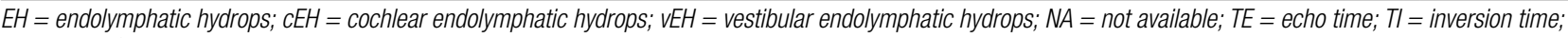
$T R=$ time of repetition.

sition or read post-processed. For example, in HYDROPS images (Hybrid of the reversed image of the positive endolymph signal and native image of positive perilymph signal) the PEI is subtracted from the PPI ${ }^{10}$. HYDROPS2 is reconstructed by subtracting the MR cisternography sequence (usually 3D heavily T2-weighted Turbo spin echo sequence with variable flip-angle) from the PEI ${ }^{11}$. HYDROPS and HYDROPS2 can be multiplied for the MR cisternography image to further increase the contrast-to-noise ratio (CNR) obtaining the HYDROPS-Mi2 and HYDROPS2-Mi2 images, respectively ${ }^{12}$. Some authors suggest that the use of maximum intensity projection (MIP) reconstruction of heavily T2-weighted 3D-FLAIR represents a robust and accurate method for assessment of $\mathrm{EH}^{13}$.

Although IT-GBCA technique has a big advantage of creating a stronger perilymph signal, the IV-GBCA technique is preferred as it is less invasive, requires only four hours to complete the MR examination and allows study of both ears in the same session ${ }^{7}$.

\section{Findings for IV-GBCA administration}

The most common method for assessment of the vestibular and cochlear endolymphatic spaces was firstly described by Nakashima et al. ${ }^{14}$ (Figs. 1-3). This method assesses the vestibular endolymphatic space (VES) by calculating the ratio of the area of the VES to the entire vestibule (VES/vestibule ratio) in the axial plane and defining the vestibular EH (vEH) absent if this ratio is $<33 \%$, mild if between $33 \%$ and $50 \%$ and significant if $>50 \%$. In addition, Nakashima et al. ${ }^{14}$ evaluated the cochlear endolymphatic space looking at displacement of Reissner's membrane and defining the cochlear EH (cEH) as mild if there is a Reissner's membrane displacement with the area of the endolymphatic compartment not exceeding the area of the scala vestibule, and as significant when the endolymphatic compartment exceeds the area of the scala vestibule ${ }^{8}$. While the aforementioned diagnostic criteria for cEH are used by almost all authors, different cut-offs of the VES/vestibule ratio for the identification $\mathrm{vEH}$ were further proposed.

Mild vEH, according to Nakashima's criteria, was reported from $94 \%$ to $100 \%$ of symptomatic ears of MD patients, but also from $53 \%$ to $100 \%$ of asymptomatic ears ${ }^{15-17}$. Sano et al. detected mild vEH in 4/6 (66\%) symptomatic ears of patients with other otological diseases ${ }^{15}$. Attyè et al. identified at least mild vEH in 27/30 (90\%) ears of healthy volunteers ${ }^{18}$. These data suggest that a VES/vestibule ratio $>33 \%$ has a low specificity in identifying the 
Table II. MR findings for IT-GBCA technique.

\begin{tabular}{|c|c|c|c|c|c|c|}
\hline Articles & $\begin{array}{l}\text { MR sequence } \\
\text { (TR/TI/TE) }\end{array}$ & $\begin{array}{c}\text { Reference MR criteria } \\
\text { for the assessment } \\
\text { of EH }\end{array}$ & $\begin{array}{l}\text { Percentage }(\%) \\
\text { of EH } \\
\text { in symptomatic } \\
\text { MD ear }\end{array}$ & $\begin{array}{l}\text { Percentage (\%) } \\
\text { of EH } \\
\text { in asymptomatic } \\
\text { MD ear }\end{array}$ & $\begin{array}{l}\text { Percentage (\%) } \\
\text { of EH in ears with } \\
\text { other audiological } \\
\text { disorders }\end{array}$ & $\begin{array}{c}\text { Percentage (\%) } \\
\text { of EH } \\
\text { in healthy ears }\end{array}$ \\
\hline $\begin{array}{l}\text { Hornibrook } \\
\text { et al. }{ }^{29}\end{array}$ & $\begin{array}{l}\text { 3D-FLAIR } \\
(\text { TI 2500) }\end{array}$ & $\begin{array}{l}\text { VEH if VES/vestibule }>33 \% \\
\text { cEH if cochlear duct is dilatated }\end{array}$ & EH: 14/30 (47\%) & NA & EH: 3/45 (7\%) & NA \\
\hline $\begin{array}{l}\text { Wu } \\
\text { et al. }{ }^{25}\end{array}$ & $\begin{array}{c}\text { 3D-FLAIR } \\
(6000 / 2100 / 387)\end{array}$ & $\begin{array}{c}\text { VEH if VES/vestibule }>50 \% \\
\text { cEH: if cochlear duct is dilatated }\end{array}$ & vEH: 75/108 (69\%) & $\begin{array}{l}\text { vEH: } 1 / 108 \\
\text { cEH: } 9 / 108\end{array}$ & NA & NA \\
\hline $\begin{array}{l}\text { Claes } \\
\text { et al. }{ }^{24}\end{array}$ & $\begin{array}{c}\text { 3D-FLAIR } \\
(9000 / 1700 / 134)\end{array}$ & $\begin{array}{l}\text { VEH if VES/vestibule }>33 \% \\
\text { cEH if cochlear duct is dilatated }\end{array}$ & $\begin{array}{l}\text { vEH 2/12 (16\%) } \\
\text { cEH: } 3 / 12(25 \%)\end{array}$ & NA & NA & NA \\
\hline $\begin{array}{l}\text { Bykowski } \\
\text { et al. }{ }^{30}\end{array}$ & $\begin{array}{c}\text { 2D-FLAIR } \\
(9454 / 2500 / 122)\end{array}$ & $\begin{array}{l}\text { vEH if VES/vestibule }>33 \% \\
\text { cEH if cochlear duct is dilatated }\end{array}$ & $\begin{array}{l}\text { vEH 6/6 (100\% } \\
\text { cEH: } 6 / 6(100 \%)\end{array}$ & NA & NA & NA \\
\hline $\begin{array}{l}\text { Naganawa } \\
\text { et al. }{ }^{27}\end{array}$ & $\begin{array}{c}\text { 3D-FLAIR } \\
(9000 / 2250 / 544)\end{array}$ & $\begin{array}{c}\text { VEH if VES/vestibule > 33\% } \\
\text { cEH: note reported }\end{array}$ & $\begin{array}{l}\text { vEH: } 8 / 9(89 \%) \\
\text { cEH: 6/9 (67\%) }\end{array}$ & $\begin{array}{l}\text { vEH: } 5 / 9(55 \%) \\
\text { cEH: } 4 / 9(44 \%)\end{array}$ & NA & NA \\
\hline $\begin{array}{l}\text { Lida } \\
\text { et al. }{ }^{26}\end{array}$ & $\begin{array}{c}\text { 3D-FLAIR } \\
(9000 / 2500 / 130)\end{array}$ & $\begin{array}{l}\text { VEH if VES/vestibule }>33 \% \\
\text { cEH if cochlear duct is dilatated }\end{array}$ & $\begin{array}{l}\text { vEH: } 9 / 11(81 \%) \\
\text { cEH:9/11 (81\%) }\end{array}$ & $\begin{array}{l}\text { vEH; 6/9 (67\%) } \\
\text { cEH: 6/9 (67\%) }\end{array}$ & NA & NA \\
\hline $\begin{array}{l}\text { Shi } \\
\text { et al. }{ }^{23}\end{array}$ & $\begin{array}{c}\text { 3D-FLAIR } \\
(9000 / 2500 / 128)\end{array}$ & $\begin{array}{l}\text { VEH if VES/vestibule }>33 \% \\
\text { cEH if cochlear duct is dilatated }\end{array}$ & EH: $3 / 4(75 \%)$ & NA & NA & NA \\
\hline $\begin{array}{l}\text { Suga } \\
\text { et al. }{ }^{28}\end{array}$ & $\begin{array}{l}\text { 3D-FLAIR } \\
\text { (NA) }\end{array}$ & $\begin{array}{l}\text { VEH if VES/vestibule }>33 \% \\
\text { cEH if cochlear duct is dilatated }\end{array}$ & $\begin{array}{l}\text { vEH 4/6 (67\%) } \\
\text { cEH 4/6 (67\%) }\end{array}$ & $\begin{array}{l}\text { VEH 1/1 (100\%) } \\
\text { cEH 1/1 (100\%) }\end{array}$ & & \\
\hline
\end{tabular}

EH = endolymphatic hydrops; $C E H=$ cochlear endolymphatic hydrops; $v E H=$ vestibular endolymphatic hydrops; $N A$ = not available; $T E=e c h o$ time; $T I=$ inversion time; $T R=$ time of repetition.

affected ear of MD patients with unilateral symptoms and in differentiating MD from other otological diseases in which a secondary vEH can occur. However, this cut-off could represent a rule out factor in MD patients in which a define diagnosis has not been clinically reached, due to its high sensitivity.

Severe vEH, according to Nakashima's criteria, was found in $46 \%$ and $68 \%$ of the symptomatic MD ears in two stud-

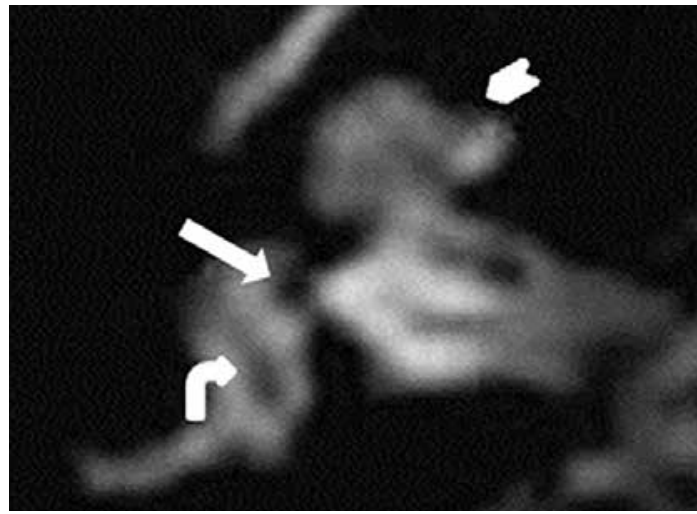

a)

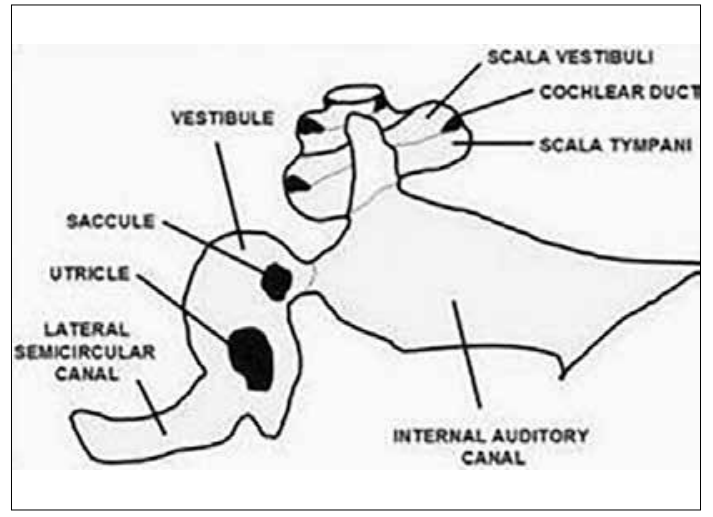

b)

Fig. 1. a) and b) T2-weighted FLAIR and schematic illustration image depicting the normal appearance of the vestibular endolymphatic space: the saccule (straight arrow) and the utricule (curved arrow) occupy less than 33\% of the vestibular space (VES/vestibule ratio); there is no enlargement of endolymphatic space in the cochlea. 


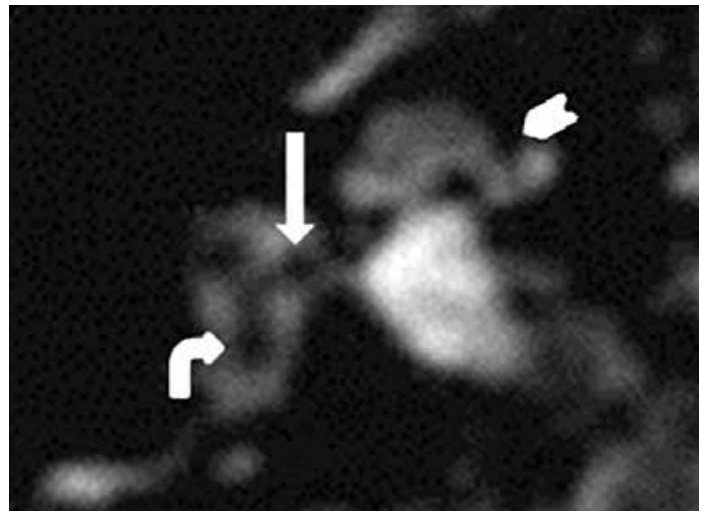

a)

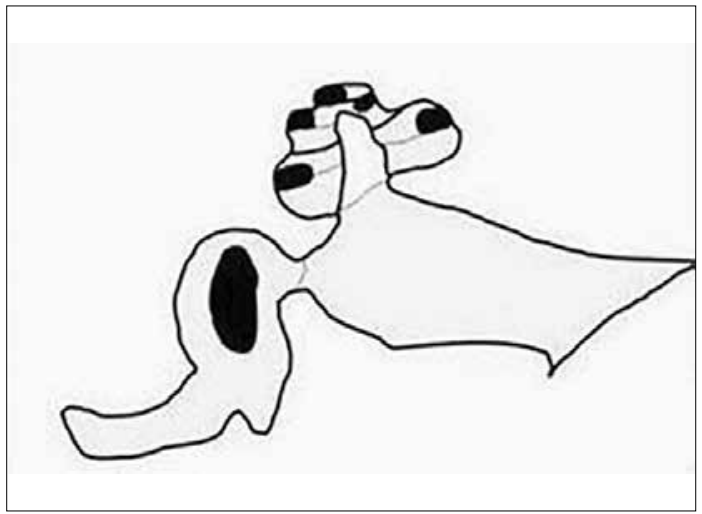

b)

Fig. 2. a) and b) T2-weighted FLAIR and schematic illustration of the endolymphatic space image demonstrate the presence of mild vEH, with a VES/vestibule ratio $>33 \%$ (straight arrow points to saccule; curved arrow points to utricle); there is also mild cEH, represented by displacement of the Reissner's membrane with the area of the endolymphatic compartment not exceeding the area of the scala vestibule (arrow-head).

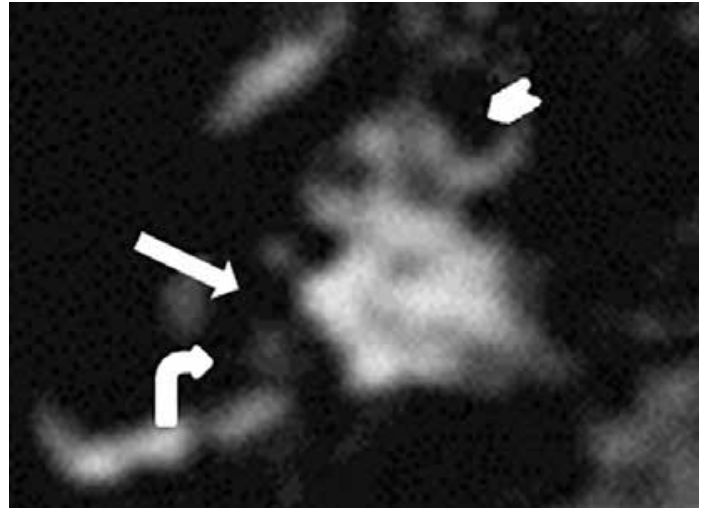

a)

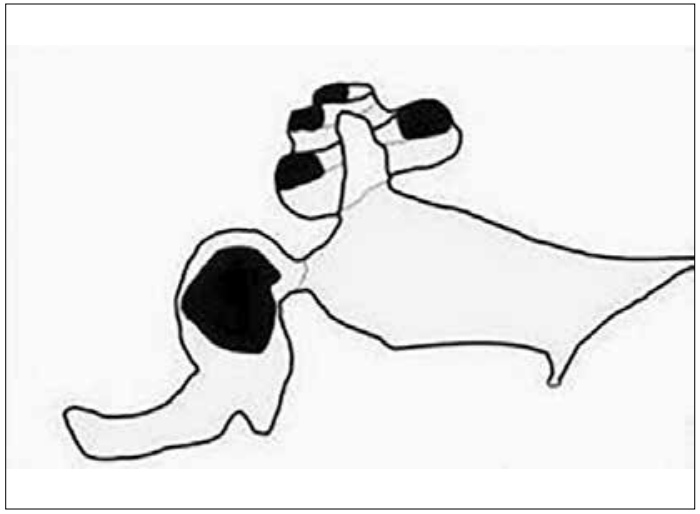

b)

Fig. 3. a) and b) T2-weighted FLAIR and schematic illustrations of the endolymphatic space image show both significant vEH and cEH. VES/vestibule ratio is $>50 \%$ (straight arrow points to saccule; curved arrow points to utricle) and the endolymphatic compartment exceeds the area of the scala vestibuli (arrow-head).

ies ${ }^{18} 19$. However, Pakdaman et al. did not find it in 32 asymptomatic ears of MD patients ${ }^{19}$. Furthermore, vEH was reported in a range from $0 \%$ to $30 \%$ of asymptomatic ears of healthy volunteers ${ }^{18} 19$. Severe vEH was identified in $15 / 128(11 \%)$ and $0 / 11(0 \%)$ symptomatic ears of patients with other otological diseases, respectively ${ }^{1920}$.

Although the VES/vestibule ratio $>50 \%$ may have a low- er sensitivity to detect MD ears, these data suggest that it may represent a rule in criteria for $\mathrm{MD}$, since it has a good specificity in both differentiating MD ears from healthy ears and those affected by other otological disorders.

Other potential cut-off values were investigated. Sepahdari et al. calculated a VES/vestibule ratio of $45 \%$ as two standard deviations above the mean of a group of patients 
with sensorineural hearing loss ${ }^{13}$. Using this cut-off value, they found $v E H$ in $6 / 12$ of the symptomatic ears of MD patients $(50 \%)$ suggesting that this diagnostic criterion cannot be used to exclude a form of MD presenting with sudden hearing loss ${ }^{13}$. Yoshida et al. reported that a VES/vestibule cut-off value of $41.9 \%$, calculated by the ROC curve, has a sensitivity of $88.5 \%$ and a specificity of $100 \%$ in differentiating MD from healthy ears ${ }^{16}$.

According to Nakashima's criteria, cEH was found in $36 \%$ $86 \%$ of symptomatic ears in three large cohorts of MD patients ${ }^{16-18}$. Yoshida et al. also assessed asymptomatic ears of both MD patients and healthy volunteers, detecting $\mathrm{cEH}$ in $46 \%$ and $33 \%$ of them, respectively ${ }^{16}$. Attyè et al. found cEH in $13 \%$ of healthy volunteers ${ }^{18}$. The latter research group found $\mathrm{cEH}$ in $24 \%$ of ears of patients with recurrent peripheral vestibulopathy ${ }^{20}$. These data suggest that $\mathrm{cEH}$, as defined by Nakashima's criteria, cannot be used to rule out MD, and can be found in approximately up to one-third of patients with other otological disease of otherwise healthy subjects, resulting less specific and preventing radiologists from diagnosing MD without the support of clinical data.

EH according to Nakashima's, either vEH (VES/vestibule ratio $>33 \%$ ) or $\mathrm{cEH}$, was reported in all 60 ears (30 MD patients and 30 healthy subjects) studied by Attyè et al., giving a sensitivity of $100 \%$ but a specificity of $0 \%{ }^{18}$. In contrast EH, defined by the presence of either vEH (VES/ vestibule ratio $>50 \%$ ) or $\mathrm{CEH}$, was reported in $77 \%-100 \%$ of symptomatic ears of MD patients 18,24,25, in 0\%-22\% of asymptomatic ears $(14,20-22)$, and in $24 \%$ of ears of patients with recurrent peripheral vestibulopathy ${ }^{20}$. This means that the presence of $\mathrm{EH}$, independently from the definition of $\mathrm{vEH}$, could be a good criterion/parameter for detecting the affected side of MD patients with unilateral symptoms, but it does not allow radiologists to differentiate MD from other otological disorders. In addition, $\mathrm{EH}$ often is not a pathologic finding, having been reported in a large number of healthy subjects.

More recently, another research group assessed hydrops using a saccular morphology-based method. The authors defined saccular hydrops as a saccule to utricle ratio (SURI) $\geq 1$, reaching a sensitivity of $50 \%$ (15/30 patients) and a specificity of $100 \%$ in differentiating the symptomatic ears of patients with Ménière's disease from the asymptomatic ears of 30 healthy volunteers ${ }^{18}$. However, it still remains unclear what the diagnostic role of this method is in differentiating MD from other otologic disorders.

\section{Findings for IT-GBCA administration}

When studying MD patients by IT-GBCA administration, Nakashima's method to investigate and assess the vestibu- lar and cochlear endolymphatic spaces should be used, as it represents a suitable and reliable method ${ }^{14}$.

Based on the common knowledge of drugs entry through an oval window pathway in rats, Shi et al. were among the first able to demonstrate a compromised passage through the oval window, showing vEH in 3 of 4 patients with definite MD ${ }^{23}$. Claes et al., in contrast, did not find any added value from the use of the IT method in evaluating the presence of EH after the injection of GBCA after a surgical procedure: the presence of $\mathrm{cEH}$ and $\mathrm{vEH}$ was demonstrated in a small percentage of patients $(25 \%$ and $16 \%$ respectively) ${ }^{24}$. A possible explanation for this low rate of positive findings can be found in the dilution factor of the administered GBCA. IT administration is still off-label and many patients are reluctant to receive a puncture to the tympanic membrane, so that unilateral IT injection is usually performed even in cases when bilateral EH is suspected, underestimating the possible involvement of the contralateral ear. The study from $\mathrm{Wu}$ et al. is one of the few in which MD patients underwent to a bilateral IT administration: presence of $\mathrm{vEH}$ was found in 75 of 108 symptomatic ears $(69 \%)$ and found $\mathrm{cEH}$ in 9 of 108 contralateral ears $(8 \%)^{25}$. The presence of EH in the contralateral asymptomatic ears was demonstrated by Lida et al. by using both IT and IV administration: $67 \%$ of asymptomatic ears were shown to have both $\mathrm{vEH}$ and $\mathrm{cEH}^{26}$. A comparison between the results obtained after simultaneous IV-IT administration was made by Naganawa et al., by using 3D-real IR images for IT-IV side and HYDROPS2 for IV ${ }^{27}$. Only HYDROPS images were able to demonstrate EH in all ears VEH and cEH were demonstrated in $89 \%$ and $67 \%$ of ears, respectively. A significant rate of $\mathrm{vEH}(55 \%)$ and $\mathrm{cEH}(44 \%)$ was also found in the contralateral asymptomatic ears ${ }^{27}$.

Differences may also exist between the times of delayed postcontrast imaging, most likely being performed after 24 hours, but in some cases after 4 hours ${ }^{28}$. The impact of this discrepancy on the imaging evaluation of MD patients is unclear: Suga et al. were able to assess the presence of vEH in 4 of 6 patients with definite MD independently from the time of acquisition ${ }^{28}$. The presence of $\mathrm{EH}$ in other audiological disorders can be shown by IT administration: Hornibrook et al. assessed the presence of EH not only in patients with definite MD (14/30 ears), but also in patients with other audiological disorders (3/45 ears), even if at a very low rate $(6 \%)^{29}$. To address potential pitfalls in the acquisition or in the interpretation of the images, Bykowski et al. used an 8 channel surface coils and acquired MR images 26 hours after monolateral IT administration in 6 definite MD patients ${ }^{30}$. Variable FLAIR inversion time images were used to determine the 
impact on fluid-suppression interpretation. $100 \%$ of definite MD patients (6/6) showed both vEH and $\mathrm{cEH}$ in the symptomatic ears.

\section{Conclusions}

MR imaging of the endolabyrithine space can be easily performed using a 4 hour-delayed volumetric acquisition after IV administration of GBCA. Thus, IT administration of GBCA, which is more invasive, is not recommended. Recent achievements in this technique have allowed radiologists to detect cochlear or vestibular $\mathrm{EH}$ on MR imaging. However, neuroimaging evidence from the literature and pathological findings described in cadavers suggest that EH does not represent exclusive findings of MD patients and is probably not always pathological, since it has been often described in normal ears. For this reason, quantitative MR assessment is most likely not sufficient in diagnosis of MD, and morphology-based criteria should be investigated. In line with this view, the more recent SURI method, described by Attyè et al. ${ }^{18}$ represents a promising tool in differentiating MD ears from ears affected by other pathologies, but further studies should investigate this method and confirm its accuracy. Along with this, it is mandatory to use only a 3 Tesla MR in the diagnostic workup of MD. We are still far from being able to use MR imaging as a new diagnostic tool for MD and its role remains mainly to exclude other diseases when clinical manifestations are not clear, and a definitive diagnosis of MD has not been reached.

\section{References}

1 Gürkov R, Pyykö I, Zou J, et al. What is Ménière's disease? A contemporary re-evaluation of EH. J Neurol 2016;263:S71-81

2 Merchant SN, Rauch SD, Nadol JB Jr. Ménière's disease. Eur Arch Otorhinolaryngol 1995; 252:63-75.

3 Lopez-Escamez JA, Carey J, Chung WH, et al. Diagnostic criteria for Ménière's disease. J Vestib Res 2015;25:1-7.

4 Merchant SN, Adams JC, Nadol JB. Pathophysiology of Ménière's syndrome are symptoms caused by EH? Otol Neurotol 2005;26:74-81.

5 Carfrae MJ, Holtzman A, Eames F, et al. 3 Tesla delayed contrast magnetic resonance imaging evaluation of Ménière's disease. Laryngoscope 2008;118:501-5.

6 Fiorino F, Pizzini FB, Beltramello A, et al. Reliability of magnetic resonance imaging performed after intratympanic administration of gadolinium in the identification of endolymphatic hydrops in patients with Ménière's disease. Otol Neurotol 2011;32:472-7.
7 Naganawa S, Nakashima T. Visualization of EH with MR imaging in patients with Ménière's disease and related pathologies: current status of its methods and clinical significance. Jpn J Radiol 2014;32:191-204.

8 Naganawa S, Sugiura M, Kawamura M, et al. Imaging of endolymphatic and perilymphatic fluid at $3 T$ after intratympanic administration of gadolinium-diethylenetriamine pentaacetic acid. AJNR Am J Neuroradiol 2008;29:724-6.

9 Naganawa S, Satake H, Kawamura M, et al. Separate visualization of endolymphatic space, perilymphatic space and bone by a single pulse sequence; $3 D$-inversion recovery imaging utilizing real reconstruction after intratympanic Gd-DTPA administration at 3 Tesla. Eur Radiol 2008;18:920-4.

10 Naganawa S, Yamazaki M, Kawai H, et al. Contrast enhancement of the anterior eye segment and subarachnoid space: detection in the normal state by heavily T2-weighted 3D FLAIR. Magn Reson Med Sci 2011;10:193-9.

11 Naganawa S, Yamazaki M, Kawai $\mathrm{H}$, et al. Imaging of Ménière's disease by subtraction of MR cisternography from positive perilymph image. Magn Reson Med Sci 2012;11:303-9.

12 Naganawa S, Yamazaki M, Kawai H, et al. Imaging of Ménière's disease after intravenous administration of single-dose gadodiamide: utility of multiplication of $M R$ cisternography and HYDROPS image. Magn Reson Med Sci 2013;12:63-8.

13 Sepahdari AR, Ishiyama G, Vorasubin N, et al. Delayed intravenous contrast-enhanced 3D FLAIR MRI in Ménière's disease: correlation of quantitative measures of endolymphatic hydrops with hearing. Clin Imaging 201;39:26-31.

14 Nakashima T, Naganawa S, Sugiura M, et al. Visualization of EH in patients with Ménière's disease. Laryngoscope 2007;415-20.

15 Sano R, Teranishi M, Yamazaki M, et al. Contrast enhancement of the inner ear in magnetic resonance images taken at 10 minutes or 4 hours after intravenous gadolinium injection. Acta Otolaryngol 2012;132:241-6.

16 Yoshida T, Sugimoto S, Teranishi M, et al. Imaging of the endolymphatic space in patients with Ménière's disease. Auris Nasus Larynx 2017;27:30142-6.

17 Tagaya M, Yamazaki M, Teranishi M, et al. Endolymphatic hydrops and blood-labyrinth barrier in Ménière's disease. Acta Otolaryngol 2011;131:474-9.

18 Attyé A, Eliezer M, Boudiaf N, et al. MRI of endolymphatic hydrops in patients with Ménière's disease: a casecontrolled study with a simplified classification based on saccular morphology. Eur Radiol 2017;27:3138-46.

19 Pakdaman MN, Ishiyama G, Ishiyama A, et al. Bloodlabyrinth barrier permeability in Ménière's disease and idiopathic sudden sensorineural hearing loss: findings on delayed postcontrast 3D-FLAIR MRI. AJNR AJNR Am J Neuroradiol 2016 Jun 2 [Epub ahead of print].

20 Attyé A, Dumas G, Troprès I, et al. Recurrent periph- 
eral vestibulopathy: is MRI useful for the diagnosis of endolymphatic hydrops in clinical practice? Eur Radiol 2015;25:3043-9.

21 Baráth K, Schuknecht B, Naldi AM, et al. Detection and grading of endolymphatic hydrops in Ménière's disease using MR imaging. AJNR Am J Neuroradiol 2014;35:1387-92.

22 Sepahdari AR, Vorasubin N, Ishiyama G, et al. Endolymphatic hydrops reversal following acetazolamide therapy: demonstration with delayed intravenous contrast-enhanced 3D-FLAIR MRI. AJNR Am J Neuroradiol 2016;37:151-4.

23 Shi H, Li Y, Yin S, et al. The predominant vestibular uptake of gadolinium through the oval window pathway is compromised by endolymphatic hydrops in Ménière's disease. Otol Neurotol 2014;35:315-22.

24 Claes G, Van den Hauwe L, Wuyts F, et al. Does intratympanic gadolinium injection predict efficacy of gentamicin partial chemolabyrinthectomy in Ménière's disease patients? Eur Arch Otorhinolaryngol 2012;269:413-8.

$25 \mathrm{Wu} \mathrm{Q}$, Dai C, Zhao M, et al. The correlation between symptoms of definite Meniere's disease and endolymphat- ic hydrops visualized by magnetic resonance imaging. Laryngoscope 2016;126:974-9.

26 Lida T, Teranishi M, Yoshida T, et al. Magnetic resonance imaging of inner ear after both intratympanic and intravenous gadolinium injections. Acta Otolaryngol 2013;133:434-8.

27 Naganawa S, Yamazaki M, Kawai $\mathrm{H}$, et al. MR imaging of Ménière's disease after combined intratympanic and intravenous injection of gadolinium using HYDROPS2. Magn Reson Med Sci 2014;13:133-7.

28 Suga K, Kato M, Yoshida T, et al. Changes in endolymphatic hydrops in patients with Ménière's disease treated conservatively for more than 1 year. Acta Otolaryngol 2015;135:866-70.

29 Hornibrook J, Flook E, Greig S, et al. MRI inner ear imaging and tone burst electrocochleography in the diagnosis of Ménière's disease. Otol Neurotol 2015;36:1109-14.

30 Bykowski J, Harris JP, Miller M, et al. Intratympanic contrast in the evaluation of Ménière's disease: understanding the limits. AJNR Am J Neuroradiol 2015;36:1326-32.

Received: November 1, 2017 - Accepted: February 1, 2018

Address for correspondence: Sonia Calloni, Postgraduation School in Radiodiagnostics, Università degli Studi di Milano, via Francesco Sforza 35, 20135 Milan, Italy. Tel. +39 02 5503.5544. E-mail: sonia.callons@gmail.com 